\title{
Allergic Bronchopulmonary Aspergillosis, Allergic Aspergillus Sinusitis and their Co-occurrence
}

\author{
Amit Diwakar, Chandramani Panjabi and Ashok Shah* \\ Department of Respiratory Medicine, Vallabhbhai Patel Chest Institute, University of Delhi, P.O. Box 2101, \\ Delhi 110 007, India
}

\begin{abstract}
Allergic bronchopulmonary aspergillosis (ABPA), which requires a set of criteria for diagnosis, occurs in atopic individuals, predominantly asthmatics. Oral corticosteroids are the cornerstone for the management of the disease. Allergic Aspergillus sinusitis (AAS), clinico-pathologically similar to ABPA, is also diagnosed with a set of criteria including demonstration of fungal elements in sinus material. Heterogeneous densities on computed tomography of the para-nasal sinuses are caused by the 'allergic mucin' in the sinuses. A combination of oral corticosteroids and surgical removal of impacted sinus mucin is the current approach to treatment. Despite common clinico-immunopathological characteristics, the co-occurrence of both these diseases is a rarely reported phenomenon. This could be due to the fact that the two diseases are often encountered by different specialities. Screening all asthmatics for Aspergillus sensitisation could identify those with severe disease and those at risk for developing ABPA. AAS must be excluded in all patients with $\mathrm{ABPA}$ and vice-versa.
\end{abstract}

Keywords: Allergic Aspergillus sinusitis, allergic bronchopulmonary aspergillosis, Aspergillus, asthma, respiratory fungi.

\section{INTRODUCTION}

Aspergillus, a ubiquitous mould, causes disease, both in the healthy and immunocompromised subjects, with diverse clinical manifestations. The three main clinical categories that are well identified are allergic aspergillosis, invasive disease and saprophytic colonisation [1]. Most commonly acquired through the inhalational route, the spores and fungal fragments lodge in the airways, both upper and lower, to cause an array of respiratory disorders (Table 1). Allergic bronchopulmonary aspergillosis (ABPA), predominantly occurring in asthmatics, was first described by Hinson and colleagues [2] in 1952, and its identity has been well established over the past six decades with reports from around the globe [3]. In 1981, Millar and colleagues [4] described the first case of allergic aspergillosis of the maxillary sinuses. Subsequently known as allergic Aspergillus sinusitis (AAS) [5], its clinico-pathological similarity to ABPA was recognised. The prevalence of concurrent ABPA and AAS is not known. In spite of overlapping mechanisms leading to both diseases, there have been very few studies which have evaluated the presence of AAS in patients of ABPA and viceversa. However, as awareness has increased, individual and multiple case reports continue to appear. We present an overview of both ABPA and AAS, and their co-occurrence.

\section{ALLERGIC BRONCHOPULMONARY ASPERGILLOSIS}

ABPA is an immunologically mediated lung disease which usually occurs in atopic individuals and is caused by hypersensitivity to the antigen of the fungus Aspergillus,

*Address correspondence to this author at the Department of Respiratory Medicine, Vallabhbhai Patel Chest Institute, University of Delhi, P.O. Box 2101, Delhi 110 007, India; Tel: 91 - 11 - 2543 3783;

Fax: 91 - 11 -2766 7420; E-mail: ashokshah99@yahoo.com
Table 1. Aspergillus-Related Respiratory Disorders [1]

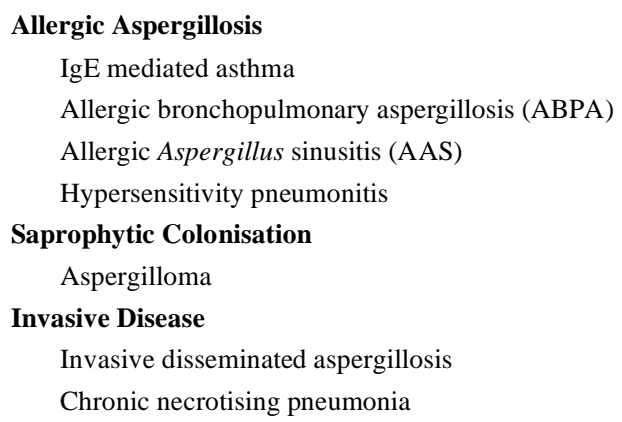

especially A. fumigutus. Repeated inhalation of fungal spores leads to airway colonisation in susceptible hosts, which evokes an allergic response. The fungal antigens commonly cause type I (IgE mediated) reaction but type III (IgGmediated immune complex) and type IV (cell mediated) responses have also been implicated. Tissue invasion, however, does not occur [6]. It affects patients with asthma and cystic fibrosis, although cases of ABPA without asthma have been reported [7, 8]. In spite of asthma running in families, familiality in ABPA is rare [9]. Among 164 patients with ABPA diagnosed over a period of 22 years in our unit, we found familial occurrence in four pairs $(4.9 \%)$. Concomitant AAS was present in one patient each in three of these four pairs [10]. The true prevalence of ABPA among asthmatics is not known, and this may be attributed to the lack of a uniform diagnostic criterion and standardised tests [11]. Reports from the USA and Europe suggest that ABPA complicates 1$6 \%$ of all chronic cases of asthma [12]. We investigated 105 patients with asthma and, of them, detected 30 (28.5\%) with a positive skin reactivity to Aspergillus antigens. Eight 
(7.6\%) of them had ABPA. The group of asthmatics with ABPA had a more severe form of the disease, as evidenced by significantly longer duration of illness, earlier age of onset of asthma as well as rhinitis, higher mean total leucocyte counts, absolute eosinophil counts and total serum IgE values. This group had significantly more number of patients with severe obstruction and had significantly more prescriptions for oral corticosteroids. Furthermore, the group of Aspergillus skin-test positive asthmatics without ABPA also had similar findings suggesting that these patients too had a more severe form of asthma as compared to those with a skin test positive to antigens other than Aspergillus [13]. Another study from India [14] reported a much higher occurrence of Aspergillus sensitisation. They found that 223 (39.5\%) of the 564 patients with asthma were sensitised and they document ABPA in $126(22.3 \%)$ of these patients. In cystic fibrosis, the prevalence of ABPA ranges from 2\% to $15 \%$ [15]. When fungi other than Aspergillus cause a similar syndrome, this is collectively known as allergic bronchopulmonary mycosis (ABPM). More than 26 different fungi have been implicated [16]. It may be difficult, without the facilities of a research laboratory, to isolate and identify the fungus responsible for ABPM. Allergic bronchopulmonary mycosis can also occur in patients with cystic fibrosis [15].

\section{DIAGNOSTIC CRITERIA}

The diagnosis of ABPA depends on a set of eight major and three minor criteria (Table 2) [17, 18]. Of these, the following five have been identified as minimal essential criteria: (1) asthma, (2) immediate cutaneous reactivity to $A$. $f u$ migatus, (3) total serum IgE $>1000 \mathrm{ng} / \mathrm{mL}$, (4) elevated specific IgE- $A f$ / IgG- $A f$, and (5) central bronchiectasis in the absence of distal bronchiectasis [15]. The occurrence of central bronchiectasis with normal tapering bronchi in ABPA was first described by Scadding [19] and is considered to be pathognomonic of the disease. However, this may not be present and the condition is then termed seropositive APBA without central bronchiectasis (ABPA-S). Considered to be an early or milder presentation of ABPA, it could, if uncontrolled, lead to ABPA with its characteristic radiological features including central bronchiectasis and, ultimately, fibrosis. Although ABPA predominantly occurs in asthmatic subjects, it can occur without clinical asthma [7, 8]. This would require a high index of suspicion and the characteristic radiological features would be of help. Pulmonary function tests are insensitive, and range from normal lung volumes with normal flow rates during periods of remission to severe obstruction with or without reversibility, and even a mixed pattern in some patients [20,21].

During the last decade or so, recombinant $A f$ allergens, having similar functional characteristics to commercial fungal extracts, have been cloned, purified, and standardised for serologic testing [22]. We investigated the immunodiagnostic relevance of synthetic epitopic peptides from the $\mathrm{N}$ terminus of Asp $\mathrm{f} 1$, an immunologically significant allergen/antigen of A. fumigatus [23]. Of the 5 peptides obtained from this potential immunodominant region, P1, an 11amino-acid synthetic peptide, inhibited the specific IgE and IgG binding of SDA (a well-defined pool of diagnostically relevant allergens and antigens of A. fumigatus). As compared to SDA, P1 also showed a higher diagnostic efficiency with ELISA. Its ability to stimulate histamine from mast cells and $\mathrm{TH}_{2}$ cytokines for ABPA patients could make it an important tool for intradermal skin testing for the diagnosis of ABPA. The utility of $\mathrm{P} 1$ as a serodiagnostic tool for ABPA in different populations around the world, as well as for other types of allergic aspergillosis, needs further evaluation. Serological testing too with different recombinant allergens helps in distinguishing patients with ABPA from Aspergillus-sensitive asthma, especially in those with cystic fibrosis [24].

Table 2. Diagnostic Criteria for Allergic Bronchopulmonary Aspergillosis [17, 18]

\begin{tabular}{|l|}
\hline Major Criteria \\
Asthma \\
Presence of transient pulmonary infiltrates (fleeting shadows) \\
Immediate cutaneous reactivity to A. fumigatus \\
Elevated total serum IgE \\
Precipitating antibodies against A. fumigatus \\
Peripheral blood eosinophilia \\
Elevated serum IgE and IgG to A. fumigatus \\
Central/proximal bronchiectasis with normal tapering of distal bron- \\
chi \\
Minor Criteria \\
Expectoration of golden brownish sputum plugs \\
Positive sputum culture for Aspergillus species \\
Late (Arthus-type) skin reactivity to A. fumigatus
\end{tabular}

\section{CLINICAL FEATURES}

An indolent disease with a protracted course, ABPA can range from mild asthma to fatal destructive lung disease. It is characterised by repeated episodes of exacerbations interspersed with periods of remissions, culminating, if untreated, in a fibrotic lung disease resembling the chronic fibrocavitary disease of pulmonary tuberculosis [1]. The vast majority of patients are asthmatics, and the most common presentation is poorly controlled asthma with peripheral eosinophilia. Apart from asthma, ABPA may also be associated with other forms of atopy, such as rhinitis, conjunctivitis, eczema, urticaria and drug as well as food allergies. Usually seen in the 20-40 years age group, ABPA has also been reported in children [25-27] and even in infants [28]. Expectoration of golden-brown plugs in the sputum is not uncommon (56\%) [29] and should raise the possibility of ABPA. However, symptoms appear to bear little or no relationship to the severity or chronicity of the disease, as a third of the patients may be relatively asymptomatic in spite of extensive radiological lesions [30].

We analysed 113 patients with ABPA, 70 of who were males [31]. The mean age of the patients was 32 years while the mean age of onset of asthma was 21 years. The mean time for diagnosis of ABPA was 11 years after the onset of asthma. Cough (99\%) and breathlessness (99\%) were the most common symptoms, followed by expectoration (98\%), wheezing (97\%) and haemoptysis (41\%). Fever was seen in $80 \%$ and nasal symptoms in 45 percent. Sputum plugs were expectorated by $37 \%$ and nasal plugs by six percent. A personal/family history of allergy was present in approximately half of the patients. 


\section{RADIOLOGICAL FEATURES}

Although ABPA has been recognised across all continents, this disease is still not diagnosed as frequently and as early as it should be [32]. Imaging of the thorax is vital to both diagnosis and monitoring the progress of the disease. The radiological manifestations are varied, and can be transient or permanent (Table 3) [1]. Consolidation or

Table 3. Radiological Changes in Allergic Bronchopulmonary Aspergillosis [1]

\begin{tabular}{|l}
\hline Transient Changes \\
Perihilar infiltrates simulating adenopathy \\
Air-fluid levels from dilated central bronchi filled with fluid and de- \\
bris \\
Massive consolidation - unilateral or bilateral \\
Radiologic infiltrates \\
'Toothpaste' shadows due to mucoid impaction in damaged bronchi \\
'Gloved finger' shadows from distally occluded bronchi filled with \\
secretions \\
'Tramline' shadows representing oedema of the bronchial walls \\
Collapse - lobar or segmental \\
Permanent Changes \\
Central bronchiectasis with normal peripheral bronchi \\
Parallel-line shadows representing bronchial widening \\
Ring-shadows 1-2 cm in diameter representing dilated bronchi en \\
face \\
Pulmonary fibrosis \\
Late changes - cavitation, contracted upper lobes and localised em- \\
physema
\end{tabular}

non-homogeneous infiltrates are commonly observed, and can disappear with or without treatment to reappear in another part of the lungs. Such 'fleeting shadows' or 'transient pulmonary infiltrates' occur due to mucoid impaction by thick bronchial secretions and parenchymal infiltrates, and are considered to be characteristic, although not pathognomic of ABPA (Figs. 1-4). Such opacities could reappear in the same site to be known as 'recurrent fixed shadows.' The involvement of upper lobes appears to be more frequent than other parts of the lungs. The demonstrations of central bronchiectasis (Fig. 5), the diagnostic radiological feature, has been made easier by computed tomography of the thorax (sensitivity of $83 \%$ and specificity of $92 \%$ ) [33], leading to the extinction of bronchography, a procedure which was invasive and risky in patients with reactive airways. Computed tomographic scans also enabled us to rapidly and safely establish the diagnosis in children with ABPA who presented with acute severe asthma [34]. The characteristic 'string of pearls' and 'signet ring' appearances are seen more commonly in the upper lobes. To date, central bronchiectasis with normal peripheral bronchi is the predominant feature and should be considered sine qua non for the diagnosis of ABPA in the absence of cystic fibrosis [35]. However, in $30 \%$ of the lobes and $21 \%$ of the segments, it has been shown to extend to the periphery [36]. There may be marked variations in the extent, type and site of bronchiectasis, even in the same patient [37]. High attenuation mucus impaction (HAM) (Figs. 6 and 7) is another radiological feature associated with ABPA and has been shown to be present in about a fourth of the patients [38] and a study [39] has linked it to initial serological severity and frequent relapses. Fibrosis, and even cavitation, can occur with the passage of time, and can present a picture similar to fibro-cavitary pulmonary tuberculosis [40, 41]. This has important clinical implications in countries where tuberculosis is prevalent, and up to $81 \%$ of patients often receive anti-tubercular treatment for long durations before arriving at the correct diagnosis of ABPA [42]. Although cavitation does occur, the occurrence of aspergilloma is surprisingly a rarity [40, 41, 43].

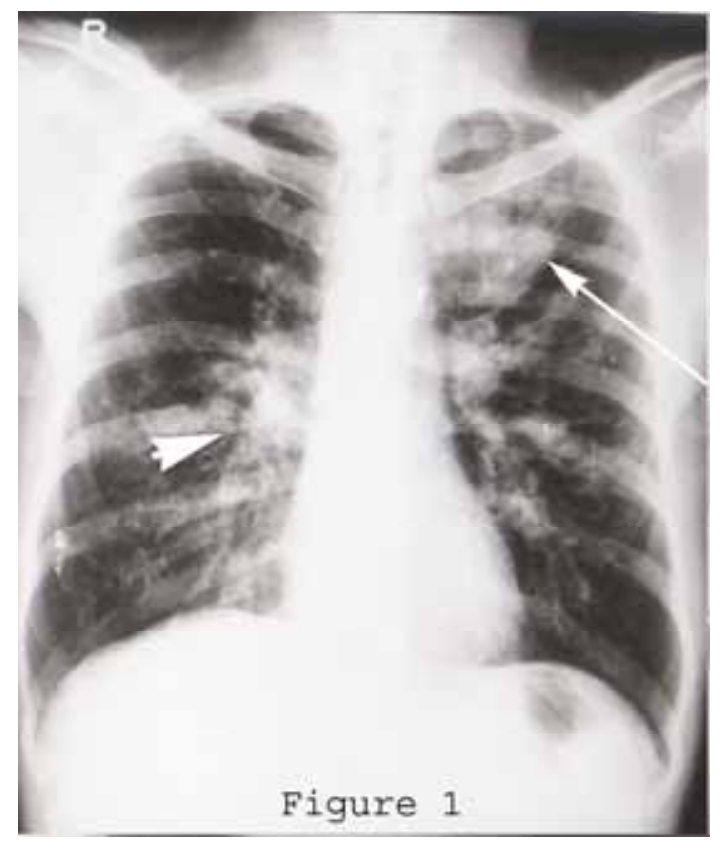

Fig. (1). Plain chest roentgenogram showing ' $\mathrm{V}-\mathrm{Y}$ ' shaped ('wine glass') (arrow) consolidation in the left upper zone along with right perihilar infiltrate (arrowhead).

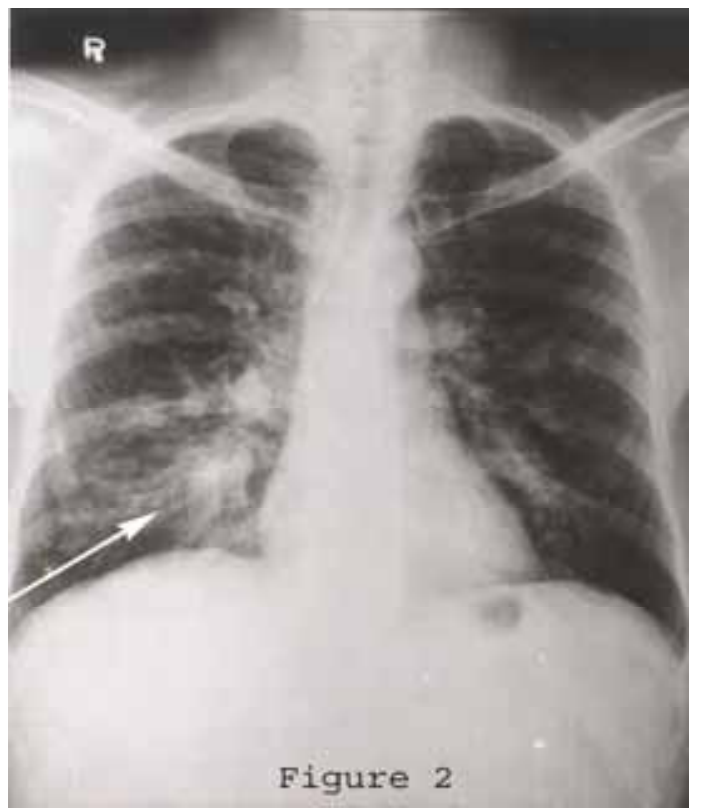

Fig. (2). Plain chest roentgenogram of the same patient taken 8 months later, showing a non homogeneous consolidation in the right lower zone (arrow). The left-sided consolidation and right sided perihilar infiltrate, seen in Fig. (1), have disappeared. 


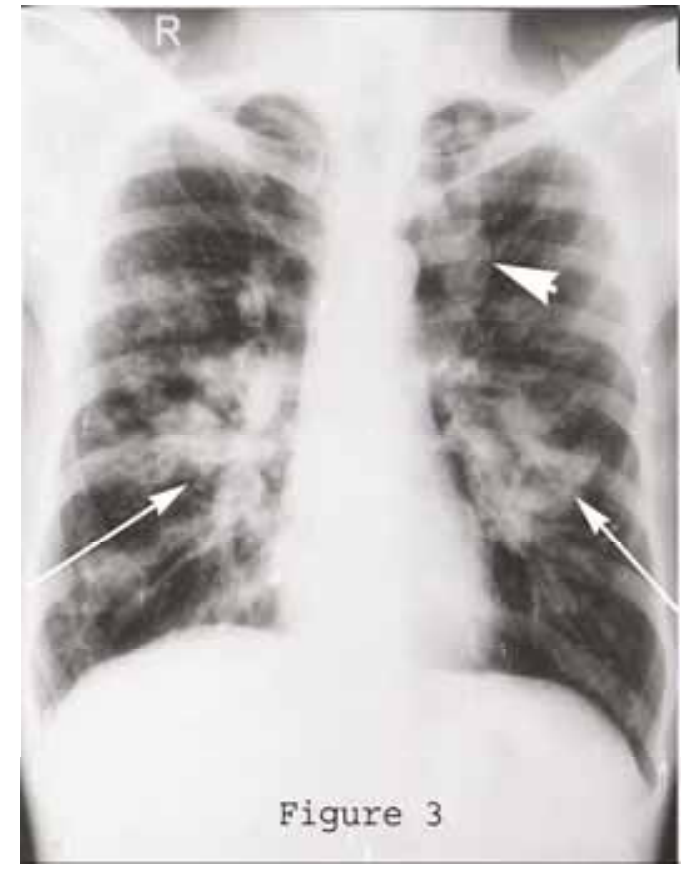

Fig. (3). Plain chest roentgenogram of the same patient taken 18 months after Fig. (1), showing re-emergence of a large consolidation in the left mid and lower zones along with right mid and lower zones consolidation (arrow). The right-sided perihilar opacity (arrow) and left upper zone consolidation have re-emerged (arrowhead).

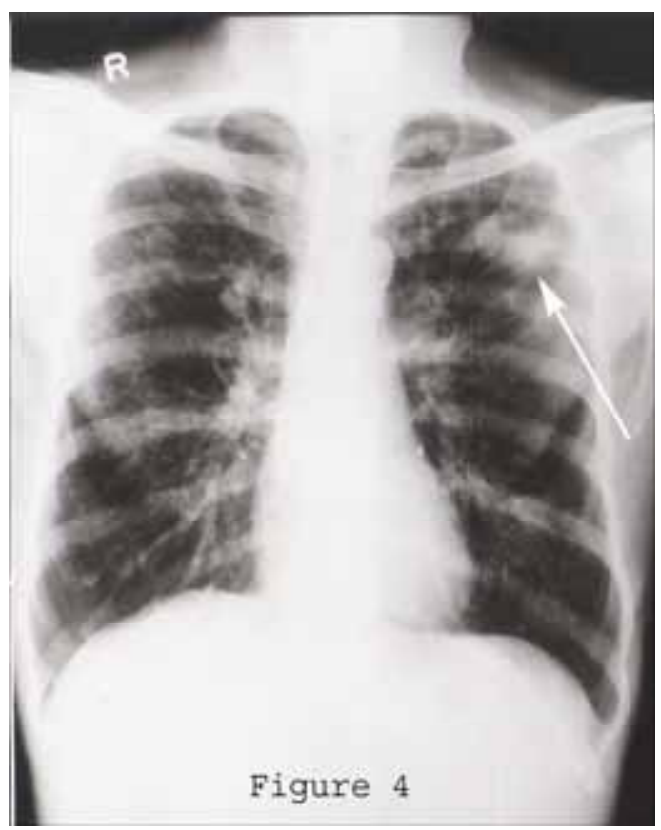

Fig. (4). Plain chest roentgenogram of the same patient taken 22 months after Fig. (1), showing that the shadows described in Fig. (3) have disappeared but a new non homogeneous consolidation has emerged in the left upper and mid zones (arrow).

Figs. (1-4) reveal 'transient pulmonary infiltrates' or 'fleeting shadows', which are characteristic of allergic bronchopulmonary aspergillosis.

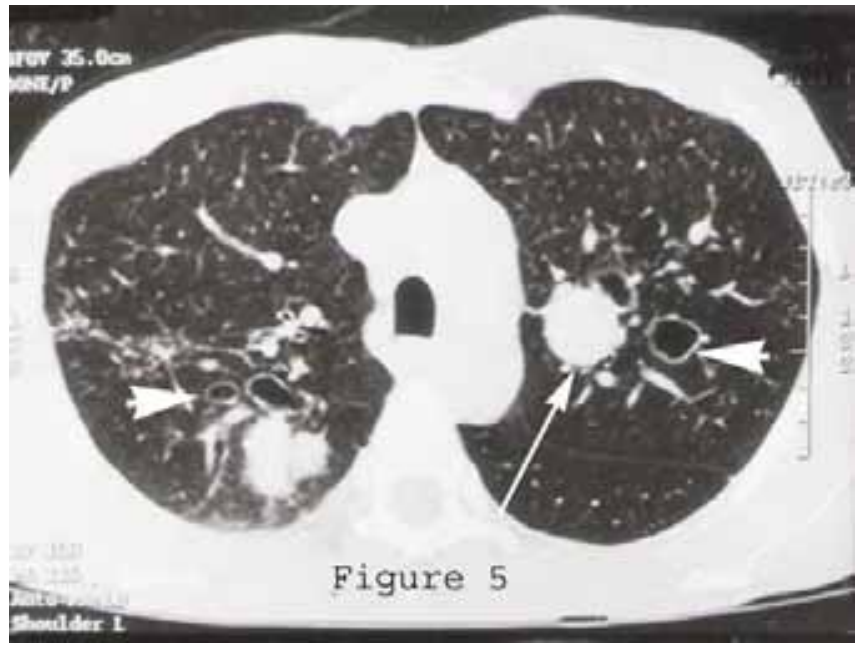

Fig. (5). HRCT scan (lung window) showing mucous-filled dilated bronchi (arrow) and extensive bilateral central bronchiectasis characterised by 'signet ring' appearance (arrowheads).
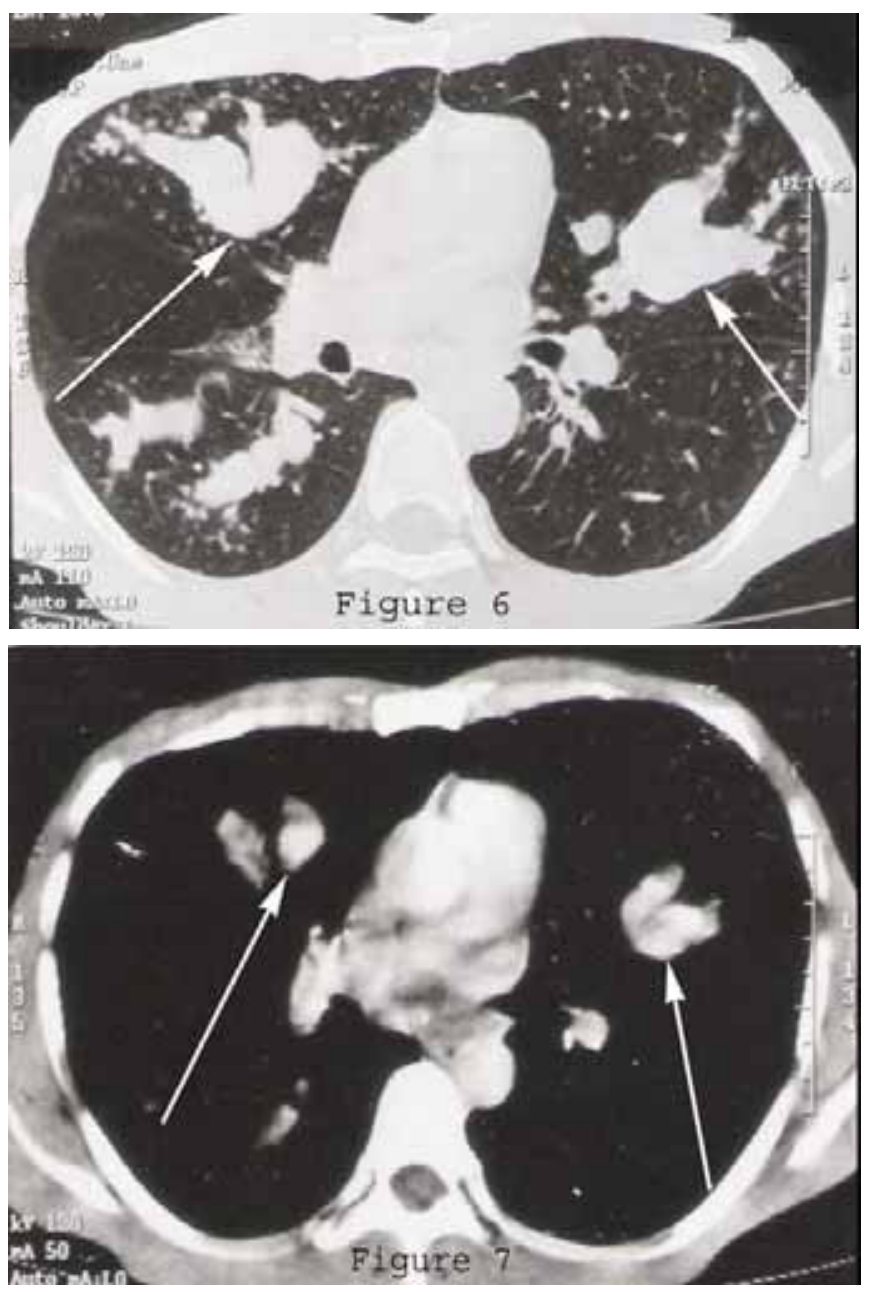

Figs. (6 and 7). HRCT scan (lung and the corresponding section on the mediastinal windows) showing high attenuation mucous (HAM) (arrows) impaction in a patient with allergic bronchopulmonary aspergillosis. 


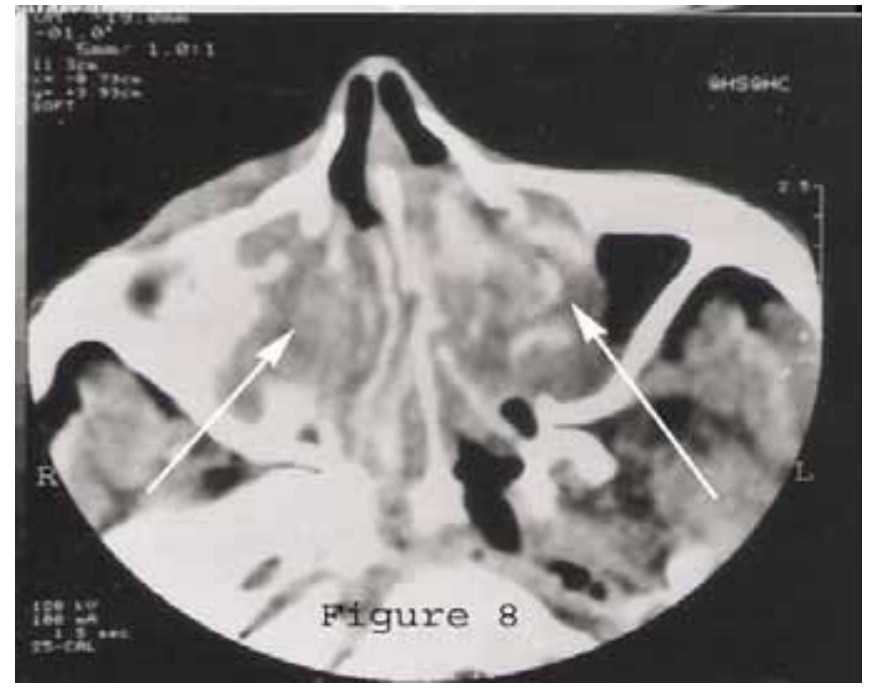

Fig. (8). Axial section of the non contrast CT- PNS showing heterogeneous densities, signifying opacification of the sinuses, with serpiginous areas of increased attenuation which are characteristic of allergic Aspergillus sinusitis (arrows).

We reviewed 1340 chest roentgenograms [31] in 113 patients of ABPA and observed fleeting shadows in $89 \%$. The other transient opacities included consolidation (91\%), perihilar infiltrates (77\%) (Fig. 1), band ('toothpaste') shadows (65\%), 'V-Y' shaped ('wine glass') shadows (27\%) (Fig. 1), 'gloved finger' opacities (23\%), lobar/segmental collapse $(17 \%)$, and air-fluid levels $(6 \%)$. True hilar adenopathy, which resolved on therapy, was seen in one patient [44]. Recently, we reported the first paediatric patient of ABPA (a 42-month-old boy) with true hilar adenopathy [27]. Concomitant occurrence of ABPA and AAS presenting as a middle lobe syndrome was also described [45]. Ipsilateral pleural effusion due to the mechanical effect of lung collapse was also reported by us in a patient of ABPA, AAS and an operated aspergilloma [46]. The effusion cleared with reexpansion of the lobe after therapy with oral corticosteroids. Other rare manifestations reported are spontaneous pneumothorax and development of broncho-pleural fistula [47].

\section{STAGING}

The 5 stages: (i) acute, (ii) remission, (iii exacerbation, (iv) corticosteroid dependent asthma, and ( $v$ ) fibrotic lung disease (Table 4) [48] were proposed mainly as an aid to management. Attempts to determine the stage should be made for every case, initially and at re-evaluation, although it is not to be presumed that the disease invariably progresses from one stage to the next in a phase-wise manner. At initial presentation, the patient is usually seen in stage III, but can also be in stage I if there is no clear-cut evidence of an earlier episode of acute disease. It is difficult to differentiate stage IV from steroid dependent asthma without ABPA, as chronic steroid treatment may mask the characteristic features of the disease [49]. Fibrotic lung disease in stage V ABPA may be associated with clubbing and cavitation [26], and may sometimes be difficult to differentiate from fibrocavitary disease caused by tuberculosis [40, 41]. Extensive bronchiectasis may resemble end stage cystic fibrosis [15].

\section{TREATMENT}

The goals of treatment of ABPA are: $(i)$ to detect and treat ABPA exacerbations promptly so as to prevent or minimise bronchiectasis that develops at the site of infiltrates, (ii) manage associated asthma (stage I to IV) or irreversible lung disease (stage V), (iii) exclude ABPA in family members, and (iv) identify a potential environmental source of the incriminated fungus [12]. No definite prognostic indicators for progression or regression of the disease have been identified. Aggressive treatment of the early stages may possibly halt progression to the stage of fibrosis [48].

Oral corticosteroids continue to remain the cornerstone of therapy over the decades. The current suggestions [15] are to administer prednisone $0.5 \mathrm{mg} / \mathrm{kg} / \mathrm{day}$ for $1-2$ weeks for new ABPA infiltrates, followed by alternate day therapy for 6-8 weeks. Attempt should then be made to discontinue prednisone by tapering by 5-10 mg every 2 weeks. The total serum $\mathrm{IgE}$ assays should be repeated in 6-8 weeks, and then every 8 weeks for 1 year to determine the range of $\mathrm{IgE}$ concentrations. However, an increase in total serum IgE of $\geq 100 \%$ over baseline can signify a silent ABPA exacerbation. However, the total serum IgE can sometimes remain elevated despite clinical and radiological improvement [42]. The chest roentgenogram or CT of the lung should be repeated after 4-8 weeks to demonstrate clearing of the infiltrates. Pulmonary function tests should be monitored. A patient who cannot be tapered off prednisone despite optimal antiasthma treatment and avoidance measures has evolved into stage IV (corticosteroid-dependent asthma) and should be managed with alternate-day prednisone as opposed to daily prednisone [15]. Concomitant conditions such as allergic rhinitis, sinusitis, and gastroesophageal reflux disease should be diagnosed and managed.

We recently assessed the feasibility of a bi-weekly prednisone regimen in 26 patients with ABPA, and found that

Table 4. Features of Five Stages of Allergic Bronchopulmonary Aspergillosis [48]

\begin{tabular}{|l|c|c|c|c|c|c|}
\hline \multicolumn{1}{|c|}{ Stage } & $\begin{array}{c}\text { Total Serum } \\
\text { IgE }\end{array}$ & $\begin{array}{c}\text { Precipitins Against } \\
\text { A.fumigatus }\end{array}$ & $\begin{array}{c}\text { Peripheral Blood } \\
\text { Eosiniphilia }\end{array}$ & $\begin{array}{c}\text { Chest Roentgenographic } \\
\text { Abnormalities }\end{array}$ & Serum IgE-Af & Serum IgG-Af \\
\hline \hline I (Acute) & +++ & + & + & + & + & + \\
\hline II (Remission) & + & \pm & + & + & \pm \\
\hline III (Exacerbation) & +++ & + & \pm & + & \pm \\
\hline $\begin{array}{l}\text { IV (Corticosteroid } \\
\text { dependent asthma) }\end{array}$ & ++ & + & - & + & \pm \\
\hline V (Fibrotic) & + & \pm & & + \\
\hline
\end{tabular}


this was as effective as alternate-day therapy in managing patients with ABPA and AAS [50]. Since oral prednisone is often given repeatedly for long periods in these patients, a bi-weekly regimen would help in reducing the well-known adverse effects of long-term corticosteroid therapy.

Inhaled corticosteroids and other anti-asthma measures may help to control the symptoms of asthma but do not prevent episodes of eosinophilic infiltration and mucus impaction. It is generally thought that these measures do not have any influence either in preventing exacerbations of ABPA or on progression of the lung damage. Patients of ABPA with rhinitis are often on long-term topical and oral steroids. We have described nasal septal perforation in one such patient [51].

The role of antifungal agents in the treatment of ABPA is still debatable. It was presumed that by decreasing the fungal load, there would be reduced antigenic stimulation and thus a decrease in the inflammatory response [52]. Itraconazole has been used as a corticosteroid-sparing agent in patients on long-term oral prednisolone [53].

A study from India [54] involving 44 patients from the Armed Forces demonstrated that itraconazole improved symptoms, lung functions, and radiologic picture. A decline in exacerbation rates too was seen. The Cochrane Database review [55] on azoles for ABPA inferred that itraconazole, in addition to modifying the immunologic activation associated with ABPA, improved clinical outcome. A subsequent review [56] concluded that, "itraconazole improves short-term symptoms and reduces the frequency of exacerbations that require the use of oral corticosteroids". The author also cautioned that the drug "may exacerbate the adrenal suppression seen with regular corticosteroids use". Until further information from large randomised, double-blind, placebocontrolled, parallel, long-term studies are available, the use of itraconazole alone should be restricted to patients in whom oral corticosteroids are absolutely contraindicated, or as an adjunct therapy. Recently, a case of ABPA with allergic fungal sinusitis was reported to be successfully treated with a one-year course of voriconazole [57].

\section{ALLERGIC ASPERGILLUS SINUSITIS}

Fungal diseases of the nose and sinuses can be invasive or non-invasive (Table 5) [58]. Allergic fungal sinusitis is now a distinct clinical entity, the central features being chronic rhinosinusitis and the presence of 'allergic mucin': eosinophil rich mucus with a peanut butter consistency observed during surgery [59]. Safirstein [60] described a case of ABPA with nasal polyposis and a history of passage of hard nasal plugs. Millar and colleagues [4] documented the first case of allergic aspergillosis of the maxillary sinuses. This condition subsequently came to be known as allergic Aspergillus sinusitis (AAS) [5]. Similarities to ABPA were noticed and a set of diagnostic criteria evolved. We have reported that one-fourth of the patients with perennial rhinitis had positive skin reactivity to Aspergillus antigens [61]. Since rhinitis is an important predisposing factor for sinusitis, these patients could possibly be at a greater risk of developing AAS. When fungi other than Aspergillus are involved, such as Alternaria, Curvularia, Bipolaris, Exserohilum, Drechslera, and Cladosporium [62-65], this is known as allergic fungal sinusitis (AFS). Five to ten percent of patients with chronic rhinosinusitis are estimated to be suffering with AFS [66-68]. Sensitisation to recombinant $A f$ allergens has demonstrated $A f$ to be an important causative mould for AFS [69].

\section{Table 5. Fungal Rhinosinusitis Disorders [58]}

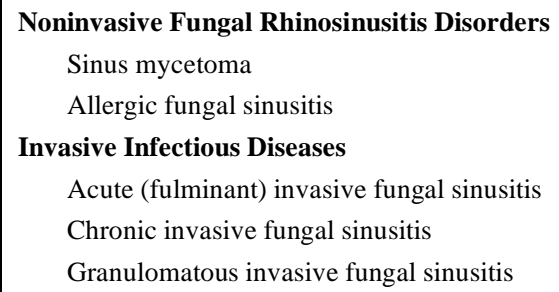

\section{CLINICAL FEATURES}

The presentation is generally subtle, and patients may give a history of rhinorrhoea, sneezing, nasal blockage, passage of nasal plugs and asthma. Nasal polyps and crusts can be found on physical examination, causing gradual obstruction of the ostia leading to accumulation of allergic mucin in the closed spaces of the sinuses. The expanding mass may cause distortion of facial features, ever so slowly that it may not be noticed. It can erode into the sinus walls to extend into the adjacent spaces such as the orbit, causing proptosis, and sometimes even loss of vision $[70,71]$. A history of several previous surgeries for recurrent sinusitis is common. Superimposed bacterial infection may lead to pain [72].

\section{DIAGNOSTIC CRITERIA}

Like ABPA, AAS is diagnosed with the help of a set of criteria. This includes radiological and histopathological features, as well as demonstration of fungal elements obtained from the sinus (Table 6) [73, 74]. Diabetics and immunocompromised patients are excluded from the diagnosis and so are patients with invasive fungal disease. Other features, akin to those in ABPA, support the diagnosis and include elevated total IgE, fungal specific IgE and IgG, cutaneous hypersensitivity to Aspergillus antigens. Precipitating antibodies to Aspergillus antigens and peripheral blood eosinophilia may also be seen [75].

\section{Table 6. Diagnostic Criteria for Allergic Aspergillus Sinusitis $[73,74]$}

Sinusitis of one or more paranasal sinus on x-ray film

Necrosed amorphous tissue along with oedematous polyps infilterated with eosinophils on histopathological evaluation of material from the sinus

Demonstration of fungal elements in nasal discharge or in material obtained at the time of surgery by stain or culture

Absence of diabetes, previous or subsequent immunodeficiency disease, and treatment with immuno-suppressive drugs

Absence of invasive fungal disease at the time of diagnosis or subsequently

Other Features [75]

Peripheral blood eosinophilia

Type I and type III cutaneous hypersensitivity to Aspergillus

Precipitating antibodies to Aspergillus antigens

Elevated total, as well as Aspergillus specific, IgE levels 


\section{RADIOLOGICAL FEATURES}

The demonstration of sinusitis on one or more paranasal sinuses on plain sinus roentgenograms is one of the diagnostic criteria for AAS. The most common manifestation is haziness of one or more sinuses. Often, multiple sinuses are opacified. Bilateral involvement has been observed in up to $83 \%$ of cases reported $[74,76]$. When sequential time related studies were performed, progressive involvement of multiple sinuses was the rule [76]. Other manifestations include mucosal thickening and varying degrees of bony sclerosis and/or destruction. These findings are, however, non specific as they may also be seen in chronic sinusitis or malignancy of the paranasal sinuses.

However, computed tomography of the paranasal sinuses has now emerged as the gold standard for evaluation of AFS. The characteristic feature is the presence of heterogeneous densities, signifying opacification of the sinuses, with serpiginous areas of increased attenuation on noncontrast scans (Fig. 8) [67, 77]. It has been speculated that metallic elements such as iron and manganese, and also salts of calcium in the allergic mucin could possibly cause such hyperdense areas [77]. The serpiginous pattern of the central area of high attenuation has also been described as 'star-filled sky' or 'ground glass' patterns [78]. The extent of disease is better visualised on $\mathrm{CT}$, and the features evaluated include bony erosion and intracranial involvement. Limited bone destruction, the exact mechanism of which is not clear, has been noted in up to $80 \%$ of patients with $\operatorname{AFS}[67,79,80]$. It has been postulated that pressure necrosis causes reabsorption of the bone or it is destroyed by enzymes produced by the fungus. Inflammatory mediators, such as major basic protein produced by the allergic response, could also be responsible [63]. This is in contrast to invasive fungal aspergillosis wherein bone destruction is evident [58]. Chronic disease of the sinuses with desiccated secretions often result in thickening and sclerosis of the adjacent sinus walls, while the presence of expansion and thinning of the sinus walls is suggestive of AFS [77]. CT also helps in distinguishing AFS from other forms of fungal sinusitis, which include acute (fulminant) invasive fungal sinusitis, chronic/indolent fungal sinusitis and sinus fungal ball [58, 81, 82]. This latter entity appears as a focal round area of increased attenuation that is usually centered within a diseased maxillary sinus. Aggressive bone erosion with extension of disease into the adjacent soft tissues is a feature of acute invasive fungal sinusitis. Unlike AFS, sinus expansion and sinus wall modeling is uncommon in acute invasive fungal sinusitis.

Magnetic resonance imaging (MRI) is now thought to be more sensitive than CT in differentiating mycotic infections of the sinuses, but it is still not considered the primary diagnostic modality [83]. Hypointense central T1 signal, central $\mathrm{T} 2$ signal void, and increased peripheral T1/T2 enhancement have been demonstrated, in 10 patients of AFS, by Manning and colleagues [84] to be highly specific for AFS. A combination of CT and MRI findings provides a radiographic appearance highly specific for AFS [85]. Its utility as a non invasive and alternative method for the diagnosis of AAS needs further evaluation.

\section{TREATMENT}

A standardised protocol for the treatment of AFS has yet to evolve. Most probably, a combined approach with surgery as well as oral corticosteroids would be required to control AFS. Surgery alone has seldom proved to be curative, and often patients have to undergo repeat procedures as recurrence is high [74]. However, it proves to be beneficial in providing drainage and aeration to the sinus mucosa, and also helps in reducing fungal load. Based on the immunopathological similarity of AAS with ABPA, treatment patterns for the former have been formulated on existing medical therapies for the latter $[74,85]$. Oral prednisone, given for long durations, has met with considerable success in prolonging recurrence after sinus surgery [86]. The optimal duration of treatment is yet to be determined. Topical corticosteroids, by themselves, are not adequate to bring relief in symptoms. Antifungals, systemic and/or topical, do not seem to have a clear cut benefit.

\section{CO-OCCURRENCE OF ALLERGIC BRONCHO- PULMONARY ASPERGILLOSIS AND ALLERGIC ASPERGILLUS SINUSITIS}

Both ABPA and AAS are IgE mediated intense inflammatory reactions of the respiratory tract occurring in atopic individuals in response to Aspergillus antigens. The allergic mucin of AFS shows histopathological similarity with the mucous plugs of ABPA, with lymphocytes, eosinophils and Charcot-Leyden crystals. The occurrence of ABPA/ABPM in some asthmatic subjects is thought to be due to the tenacious secretions within the bronchi which provide a hospitable environment for the growth of fungi and subsequent release of antigenic material. This sets into motion a host of immunological reactions culminating in pulmonary damage [9]. Since pathological similarities to ABPA/ABPM are considered to be the hallmark of AAS/AFS, it is likely that a similar chain of events may be responsible for its onset. An alternative explanation is that the changes in the bronchial or sinus mucous and the secretory immune system that favour growth of fungi may exist in bronchi as well as sinuses of patients who develop ABPA/ABPM and AAS/AFS [9]. The factors responsible for the predilection of the fungus for the sinuses in some patients and the bronchi in others remain unknown [74]. Oral corticosteroids, to date, remain the key to management of both diseases.

However, in spite of these similarities, it is perhaps surprising that their coexistence has not often been reported. Safirstein [60] first described a patient with ABPA with obstruction of the upper respiratory tract and a history of passage of hard nasal plugs. This report, in retrospect, is thought to be a case of concomitant AAS and ABPA. Sher and Schwartz [87], in 1988, published the first report of a case of AAS with concurrent ABPA. Several such cases have since been reported [9, 43, 45, 46, 57, 74, 88-92], of which eight were published by us. These included some unique presentations such as associated familial occurrence of ABPA [9], middle lobe syndrome [45], collapsed lung with pleural effusion and prior operated aspergilloma [46] and a case of coexisting aspergilloma [43]. 
In our review of 95 patients with ABPA [74], we were able to confirm the presence of concomitant AAS in seven patients of the nine patients who consented to undergo surgical procedures for obtaining histopathological specimen from the sinuses, necessary for the diagnosis of AAS. However, we had 13 other patients with radiological evidence of sinusitis in whom the possibility of AAS could not be ruled out as they refused to undergo such invasive procedures. Hypothetically, the frequency of AAS among our patients with ABPA could be higher. The availability of minimally invasive methods for procuring sinus material could help in reaching the diagnosis more easily. In 2002, Venarske and deShazo [90] coined the term "sinobronchial allergic mycosis" (the SAM syndrome) to highlight the expression of fungal hypersensitivity in both upper and lower airways.

Asthma and sinusitis are two diseases often treated by two different specialities. The occurrence of AAS in ABPA and ABPA in AAS may thus be overlooked [74]. In a series of 44 patients with AFS from the Mayo Clinic [80], there was no mention of concomitant ABPA. In another series of 67 consecutive cases of AFS predominantly caused by Bipolaris spicifera, concomitant AFS and ABPM were seen in only two patients [65]. Both these patients had been previously diagnosed with ABPM after undergoing partial lobectomy. A study on 126 patients of ABPA [14] from India does not mention any patient with nasal symptoms, or any attempt to diagnose concomitant AAS. Recently, a study on 20 children with fungal sinusitis [93] identified 11 of them with asthma, but these were not investigated further for the presence of ABPA.

\section{CONCLUSION}

The concept of "one airway one disease" [94] is further strengthened with the increase in recognition of the association between ABPA and AAS, reflecting fungal hypersensitivity in both the upper and lower airways. The true incidence of co-occurrence of both diseases is yet to be evaluated. Giving due importance to nasal symptoms in patients with ABPA, and chest symptoms in patients with AAS, could increase the diagnosis of concurrent disease, and could possibly reduce morbidity in such patients.

\section{REFERENCES}

[1] Shah A. Allergic bronchopulmonary aspergillosis. Indian J Chest Dis Allied Sci 1998; 40: 41-54.

[2] Hinson KFW, Moon AJ, Plummer NS. Bronchopulmonary aspergillosis: a review and report of eight new cases. Thorax 1952; 7 : 317-33.

[3] Shah A, Panjabi C. Allergic bronchopulmonary aspergillosis: A review of a disease with a worldwide distribution. J Asthma 2002; 39: 273-89.

[4] Millar JW, Johnston A, Lamb D. Allergic aspergillosis of the maxillary sinuses [abstract]. Thorax 1981; 36: 710 .

[5] Katzenstein AL, Sale SR, Greenberger PA. Allergic aspergillus sinusitis: a newly recognized form of sinusitis. J Allergy Clin Immunol 1983; 72: 89-93.

[6] Patterson R. Allergic bronchopulmonary aspergillosis and hypersensitivity reactions to fungi. In: Fishman AP, Elias JA, Fishman JA, Grippi MA, Kaiser CR, Senior RM, Eds. Fishman's Pulmonary Diseases and Disorders; 3rd ed, New York, McGraw-Hill \& Co. 1998; Vol. 1: pp 777-82.

[7] Glancy JJ, Elder JL, McAleer R. Allergic bronchopulmonary fungal disease without clinical asthma. Thorax 1981; 36: 345-9.

[8] Shah A, Maurya V, Panjabi C, Khanna P. Allergic bronchopulmonary aspergillosis without clinical asthma caused by Aspergillus niger. Allergy 2004; 59: 236-7.
[9] Shah A, Khan ZU, Chaturvedi S, Bazaz Malik G, Randhawa HS. Concomitant allergic Aspergillus sinusitis and allergic bronchopulmonary aspergillosis associated with familial occurrence of allergic bronchopulmonary aspergillosis. Ann Allergy 1990; 64: 507-12.

[10] Shah A, Kala J, Sahay S, Panjabi C. Frequency of familial occurrence in 164 patients with allergic bronchopulmonary aspergillosis. Ann Allergy Asthma Immunol (in press).

[11] Shah A. Allergic bronchopulmonary aspergillosis: a view from India. Saudi Med J 2002; 23: 1559-61.

[12] Greenberger PA. Allergic bronchopulmonary aspergillosis. In: Adkinson NF Jr, Yunginger JW, Busse WW, Bochner BS, Holgate ST, Simon FER, Eds. Middleton's Allergy: principles and practice, $6^{\text {th }}$ ed. Philadelphia: CV Mosby Co. 2003; pp. 1353-71.

[13] Maurya V, Gugnani HC, Sarma PU, Madan T, Shah A. Sensitization to Aspergillus antigens and occurrence of allergic bronchopulmonary aspergillosis in patients with asthma. Chest 2005; 127: $1252-9$.

[14] Agarwal R, Gupta D, Aggarwal AN, Behera D, Jindal SK. Allergic bronchopulmonary aspergillosis: lessons from 126 patients attending a chest clinic in north India. Chest 2006; 130: 442-8.

[15] Greenberger PA. Allergic bronchopulmonary aspergillosis. J Allergy Clin Immunol 2002; 110: 685-92.

[16] Patterson R. Allergic bronchopulmonary aspergillosis: a historical perspective. Immunol Allergy Clin North Am 1998; 18: 471-8.

[17] Rosenberg M, Patterson R, Mintzer R, Cooper BJ, Roberts M, Harris KE. Clinical and immunologic criteria for the diagnosis of allergic bronchopulmonary aspergillosis. Ann Intern Med 1977; 86: 405-14.

[18] Wang JLF, Patterson R, Rosenberg M, Roberts M, Cooper BJ. Serum IgE and IgG antibody activity against Aspergillus fumigatus as a diagnostic aid in allergic bronchopulmonary aspergillosis. Am Rev Respir Dis 1978; 117: 917-27.

[19] Scadding JG. The bronchi in allergic aspergillosis. Scand J Respir Dis 1967; 48: 372-7.

[20] Nichols D, DoPics GA, Braun S, Imbean S, Peters ME, Rankin J. Acute and chronic pulmonary function changes in allergic bronchopulmonary aspergillosis. Am J Med 1979; 67: 631-7.

[21] Panjabi C, Shah A. Lung functions in allergic bronchopulmonary aspergillosis. Respirology 2006; 11: A38.

[22] Crameri R, Lidholm J, Gronlund H, Stuber D, Blaser K, Menz G. Automated specific IgE assay with recombinant allergens: evaluation of the recombinant Aspergillus fumigatus allergen $\mathrm{I}$ in the Pharmacia CAP System. Clin Exp Allergy 1996; 26: 1411-9.

[23] Madan T, Priyadarsiny P, Vaid M, et al. Use of a synthetic peptide epitope of Asp f 1, a major allergen or antigen of Aspergillus fumigatus, for improved immunodiagnosis of allergic bonchopulmonary aspergillosis. Clin Diagn Lab Immunol 2004; 11: 552-8.

[24] Knutsen AP, Hutcheson PS, Slavin RG, Kurup VP. IgE antibody to Aspergillus fumigatus recombinant allergens in cystic fibrosis patients with allergic bronchopulmonary aspergillosis. Allergy 2004; 59: 198-203.

[25] Chetty A, Bhargava S, Jain RK. Allergic bronchopulmonary aspergillosis in Indian children with bronchial asthma. Ann Allergy 1985; 54: 46-9.

[26] Shah A, Bhagat R, Panchal N. Allergic bronchopulmonary aspergillosis with clubbing and cavitation. Indian Pediatr 1993; 30: 24851.

[27] Shah A, Kala J, Sahay S. Allergic bronchopulmonary aspergillosis with hilar adenopathy in a 42-month-old boy. Pediatr Pulmonol 2007; 42: 747-8.

[28] Imbeau SA, Cohen M, Reed CE. Allergic bronchopulmonary aspergillosis in infants. Am J Dis Child 1977; 131: 1127-30.

[29] McCarthy DS, Pepys J. Allergic bronchopulmonary aspergillosis. Clinical immunology: (1) clinical features. Clin Allergy 1971; 1: 261-86.

[30] Safirstein BH, D’Souza MF, Simon G, Tai EH-C, Pepys J. Fiveyear follow up of bronchopulmonary aspergillosis. Am Rev Respir Dis 1973; 108: 450-9.

[31] Shah A, Panchal N, Panjabi C. Allergic bronchopulmonary aspergillosis: a review from India [abstract]. Allergy Clin Immunol Int-J World Allergy Org 2003; (Suppl 1): 104.

[32] Shah A. Allergic bronchopulmonary aspergillosis. Allergy Clin Immunol Int 2005; 17: 172-80.

[33] Panchal N, Pant CS, Bhagat R, Shah A. Central bronchiectasis in allergic bronchopulmonary aspergillosis: comparative evaluation of 
computed tomography of the thorax with bronchography. Eur Respir J 1994; 7: 1290-3.

[34] Shah A, Pant CS, Bhagat R, Panchal N. Computed tomography in childhood allergic bronchopulomonary aspergillosis. Pediatr Radiol 1992; 22: 227-8

[35] Shah A. Allergic bronchopulmonary aspergillosis: an Indian perspective. Curr Opin Pulm Med 2007; 13: 72-80.

[36] Panchal N, Bhagat R, Pant C, Shah A. Allergic bronchopulmonary aspergillosis: the spectrum of computed tomography appearances. Respir Med 1997; 91: 213-9.

[37] Shah A. Radiological aspects of allergic bronchopulmonary aspergillosis and allergic Aspergillus sinusitis. Mold allergy, biology and pathogenesis. In: Kurup VP, Ed. Trivandrum: Research Signpost 2005; pp. 147-62.

[38] Logan PM, Muller NL. High-attenuation mucous plugging in allergic bronchopulmonary aspergillosis. Can Assoc Radiol J 1996; 47: 374-7.

[39] Agarwal R, Gupta D, Aggarwal AN, Saxena AK, Chakrabarti A, Jindal SK. Clinical significance of hyperattenuating mucoid impaction in allergic bronchopulmonary aspergillosis: an analysis of 155 patients. Chest 2007; 132: 1183-90.

[40] Shah A, Khan ZU, Chaturvedi S, Ramchandran S, Randhawa HS, Jaggi OP. Allergic bronchopulmonary aspergillosis with coexistent aspergilloma: a long term follow up. J Asthma 1989; 26: $109-15$.

[41] Agarwal AK, Bhagat R, Panchal N, Shah A. Allergic bronchopulmonary aspergillosis with aspergilloma mimicking fibrocavitory pulmonary tuberculosis. Asian Pac J Allergy Immunol 1996; 14: 58.

[42] Shah A. Aspergillus associated hypersensitivity respiratory disorders. Indian J Chest Dis Allied Sci 2008; 50: 117-28.

[43] Shah A, Panjabi C. Contemporaneous occurrence of allergic bronchopulmonary aspergillosis, allergic Aspergillus sinusitis and aspergilloma. Ann Allergy Asthma Immunol 2006; 96: 874-8.

[44] Shah A, Aggarwal AK, Chugh IM. Hilar adenopathy in allergic bronchopulmonary aspergillosis. Ann Allergy Asthma Immunol 1999; 82: 504-6.

[45] Shah A, Bhagat R, Panchal N, Jaggi OP, Khan ZU. Allergic bronchopulmonary aspergillosis with middle lobe syndrome and allergic Aspergillus sinusitis. Eur Respir J 1993; 6: 917-8.

[46] Bhagat R, Shah A, Jaggi OP, Khan ZU. Concomitant allergic bronchopulmonary aspergillosis and allergic Aspergillus sinusitis with an operated aspergilloma. J Allergy Clin Immunol 1993; 91: 10946.

[47] Judson MA, Marshall C, Beale G, Holt JB. Pneumothorax and bronchopleural fistula during treatment of allergic bronchopulmonary aspergillosis. South Med J 1993; 86: 1061-3.

[48] Patterson R, Greenberger PA, Radin RC, Roberts M. Allergic bronchopulmonary aspergillosis: staging as an aid to management. Ann Intern Med 1982; 96: 286-91.

[49] Ramachandran S, Shah A, Pant K, Bhagat R, Jaggi OP. Allergic bronchopulmonary aspergillosis and Candida albicans colonization of the respiratory tract in corticosteroid dependent asthma. Asian Pac J Allergy Immunol 1990; 8: 123-6.

[50] Shah A, Panjabi C. Biweekly therapy with prednisolone is effective in the management of allergic bronchopulmonary aspergillosis [abstract]. Allergy Clin Immunol Int-J World Allergy Org 2005; (Suppl 1): 113.

[51] Deepak D, Panjabi C, Gudwani S, Chaudhary N, Shah A. Nasal septal perforation in a patient with allergic bronchopulmonary aspergillosis and rhinitis on long term corticosteroids. Asian Pac J Allergy Immunol 2001; 19: 287-90.

[52] Malo JL. Antifungal therapy for allergic bronchopulmonary aspergillosis. J Allergy Clin Immunol 2003; 111: 934-5.

[53] Leon EE, Craig TJ. Antifungals in the treatment of allergic bronchopulmonary aspergillosis. Ann Allergy Asthma Immunol 1999; 82: 511-7.

[54] Rai SP, Panda BN, Bhargava S. Treatment of allergic bronchopulmonary aspergillosis with fluconazole and itraconazole. Med J Armed Forces India 2004; 60: 128-30.

[55] Wark PA, Gibson PG, Wilson AJ. Azoles for allergic bronchopulmonary aspergillosis associated with asthma. Cochrane Database Syst Rev 2004; 3: CD001108.

[56] Wark P. Pathogenesis of allergic bronchopulmonary aspergillosis and an evidence-based review of azoles in treatment. Respir Med 2004; 98: 915-23.
[57] Erwin GE, Fitzgerald JE. Allergic bronchopulmonary aspergillosis and allergic fungal sinusitis successfully treated with voriconazole. J Asthma 2007; 44: 891-5.

[58] deShazo RD, Chapin K, Swain RE. Fungal sinusitis. N Engl J Med 1997; 337: 254-9.

[59] Katzenstein AL, Sale SR, Greenberger PA. Pathologic findings in allergic aspergillus sinusitis: a newly recognized form of sinusitis. Am J Surg Pathol 1983; 7: 439-43.

[60] Safirstein BH. Allergic bronchopulmonary aspergillosis with obstruction of the upper respiratory tract. Chest 1976; 70: 788-90.

[61] Shah A, Sircar M. Sensitization to Aspergillus antigens in perennial rhinitis. Asian Pac J Allergy Immunol 1991; 9: 137-9.

[62] Corey JP, Delsupehe KG, Ferguson BJ. Allergic fungal sinusitis: allergic, infectious, or both? Otolaryngol Head Neck Surg 1995; 113: 110-9.

[63] Manning SC, Schaefer SD, Close LG, Vuitch F. Culture-positive allergic fungal sinusitis. Arch Otolaryngol Head Neck Surg 1991; 117: 174-8.

[64] Bartynski JM, McCaffrey TV, Frigas E. Allergic fungal sinusitis secondary to dematiaceous fungi: Curvularia lunata and Alternaria. Otolaryngol Head Neck Surg 1990; 103: 32-9.

[65] Schubert MS, Goetz DW. Evaluation and treatment of allergic fungal sinusitis. I. Demographics and diagnosis. J Allergy Clin Immunol 1998; 102: 387-94.

[66] Corey JP. Allergic fungal sinusitis. Otolaryngol Clin North Am 1992; 25: 225-30.

[67] Bent J, Kuhn F. Diagnosis of allergic fungal sinusitis. Otolaryngol Head Neck Surg 1994; 111: 580-8.

[68] Deshpande RB, Shaukla A, Kirtane MV. Allergic fungal sinusitis: incidence and clinical and pathological features of seven cases. J Assoc Physicians India 1995; 43: 98-100.

[69] McCann WA, Cromie M, Chandler F, Ford J, Dolen WK. Sensitization to recombinant Aspergillus fumigatus allergens in allergic fungal sinusitis. Ann Allergy Asthma Immunol 2002; 89: 203-8.

[70] Brummund W, Kurup VP, Harris GJ, Duncavage JA, Arkins JA Allergic sino-orbital mycosis. A clinical and immunologic study. JAMA 1986; 256: 3249-53

[71] Marple BF, Gibbs SR, Newcomer MT, Mabry RL. Allergic fungal sinusitis-induced visual loss. Am J Rhinol 1999; 13: 191-5.

[72] Marple BF. Allergic fungal sinusitis. Curr Opin Otolaryngol 1999; 7: 383-7.

[73] deShazo RD, Swain RE. Diagnostic criteria for allergic fungal sinusitis. J Allergy Clin Immunol 1995; 96: 24-35.

[74] Shah A, Panchal N, Agarwal AK. Concomitant allergic bronchopulmonary aspergillosis and allergic Aspergillus sinusitis: a review of an uncommon association. Clin Exp Allergy 2001; 31: 1896-905.

[75] Shah A. Allergic bronchopulmonary and sinus aspergillosis: the cooccurrence [editorial]. Chest (India) 2001; 2: 234-5.

[76] Waxman JE, Spector JG, Sale SR, Katzenstein A-LA. Allergic Aspergillus sinusitis: concepts in diagnosis and treatment of a new clinical entity. Laryngoscope 1987; 97: 261-6.

[77] Mukherji SK, Figueroa RE, Ginsberg LE, et al. Allergic fungal sinusitis: CT findings. Radiology 1998; 207: 417-22.

[78] Houser SM, Corey JP. Allergic fungal rhinosinusitis: pathophysiology, epidemiology and diagnosis. Otolaryngol Clinics North Am 2000; 33: 399-408.

[79] Manning SC, Vuitch F, Weinberg AG, Brown OE. Allergic aspergillosis: a newly recognized form of fungal sinusitis in the pediatric population. Laryngoscope 1989; 99: 681-5.

[80] Cody DT II, Neel HB III, Ferreiro JA, Roberts GD. Allergic fungal sinusitis: the Mayo clinic experience. Laryngoscope 1994; 104: 1074-9.

[81] Goldstein MF, Atkins PC, Cogen FC, Kornstein MJ, Levine RS, Zweiman B. Allergic Aspergillus sinusitis. J Allergy Clin Immunol 1985; 76: 515-24.

[82] Morpeth JF, Rupp NT, Dolen WK, Bent JP III, Kuhn FA. Fungal sinusitis: an update. Ann Allergy Asthma Immunol 1996; 76: 12840 .

[83] Shah A. Allergic bronchopulmonary and sinus aspergillosis: the roentgenologic spectrum. Front Biosci 2003; 8: e138-46.

[84] Manning SC, Merkel M, Kreisel K, Vuitch F, Marple B. Computed tomographic and magnetic resonance diagnosis of allergic fungal sinusitis. Laryngoscope 1997; 107: 170-6.

[85] Marple BF. Allergic fungal rhinosinusitis: current theories and management strategies. Laryngoscope 2001; 111: 1006-19. 
[86] Schubert MS, Goetz DW. Evaluation and treatment of allergic fungal sinusitis. II: treatment and follow-up. J Allergy Clin Immunol 1998; 102: 395-402.

[87] Sher TH, Schwartz HJ. Allergic Aspergillus sinusitis with concurrent allergic bronchopulmonary Aspergillus: report of a case. J Allergy Clin Immunol 1988; 81: 844-6.

[88] Schwartz HJ, Witt WJ, Sher TH. Allergic bronchopulmonary aspergillosis and allergic aspergillus sinusitis: case report. Ann Allergy 1992; 69: 447-8.

[89] Schwartz HJ. Allergic fungal sinusitis: experience in an ambulatory allergy practice. Ann Allergy Asthma Immunol 1996; 77: 500-2.

[90] Venarske DL, deShazo RD. Sinobronchial allergic mycosis: The SAM syndrome. Chest 2002; 121: 1670-6.
[91] Braun JJ, Pauli G, Schultz P, Gentine A, Ebbo D, de Blay F. Allergic fungal sinusitis associated with allergic bronchopulmonary aspergillosis: an uncommon sinobronchial allergic mycosis. Am J Rhinol 2007; 21: 412-6.

[92] Prasad R, Garg R, Sanjay, Dua R. Concomitant allergic bronchopulmonary aspergillosis and allergic Aspergillus sinusitis [letter]. Indian J Med Sci 2007; 61: 532-4.

[93] Campbell JM, Graham M, Gray HC, Bower C, Blaiss MS, Jones SM. Allergic fungal sinusitis in children. Ann Allergy Asthma Immunol 2006; 96: 286-90.

[94] Grossman J. One airway, one disease. Chest 1997; 111: 11S-16S.

(c) Diwakar et al.; Licensee Bentham Open.

This is an open access article licensed under the terms of the Creative Commons Attribution Non-Commercial License (http://creativecommons.org/licenses/bync/3.0/) which permits unrestricted, non-commercial use, distribution and reproduction in any medium, provided the work is properly cited. 